\title{
GPPS-BJ-2019-180
}

\section{AN ANALYTICAL METHOD FOR A RECTANGULAR SPLITTER SILENCER}

\author{
Lingfeng Chen* \\ Beihang University \\ chenlingfeng@buaa.edu.cn \\ Beijing, P. R. CHINA. \\ Lin Du \\ Beihang University \\ lindu@buaa.edu.cn \\ Beijing, P. R. CHINA.
}

\author{
Xiaoyu Wang \\ Beihang University \\ bhwxy@sip.buaa.edu.cn \\ Beijing, P. R. CHINA. \\ Xiaofeng Sun \\ Beihang University \\ sunxf@buaa.edu.cn \\ Beijing, P. R. CHINA.
}

\begin{abstract}
This paper presents a three-dimensional analytical method for predicting the sound attenuation performance of the porous splitter silencer commonly used in a HVAC system. This method avoids troublesome eigenvalue calculations by applying the equivalent source method and separating the sound field in the silencer into primary and secondary sound fields. The distribution of the equivalent source is represented via the trigonometric series expansion technique. The modal amplitudes of the primary sound field are finally obtained through mode matching in the inlet and outlet planes. The transmission loss of a simple rectangular splitter silencer is calculated both for plane wave and higher order mode incidence. The predicted results by using this method are in good agreement with those of the finite element method.
\end{abstract}

\section{INTRODUCTION}

The splitter silencer is commonly used to attenuate broadband noise in a HVAC (heating, ventilation and air conditioning) system which typically has a rectangular cross section (Kirby and Lawrie, 2005). The upper frequency limit of the noise can reach to as high as $8 \mathrm{kHz}$ (Kirby, 2005) and the HVAC duct size is often relatively large, which brings a significant computational difficulty in accessing the performance of such kind of silencer. Traditional analytical methods (Ko, 1975) are expected to give a fast computation tool to this problem but most methods focus on modal attenuation in an infinite duct thus neglecting important acoustic scattering effects of either end of the silencer (Kirby, 2005). In addition, calculating the eigenvalues is also another inevitable barrier in applying these methods in practice. The numerical methods (Kirby, 2005, Lawrie and Kirby, 2006) can consider a finite silencer and more complex structures such as "bull nose" fairings (Lawrie and Kirby, 2006), but the large dimension of the duct limits their ability to solve the sound field in a high frequency range. The reduced 2-dimensional method (Kirby, 2005, Kirby et al., 2014) can overcome this shortage but it requires plane wave incidence which cannot be guaranteed in splitter silencer applications.

This paper develops a new analytical method to calculate the sound attenuation level of a finite splitter silencer. For simplicity, only one porous splitter is considered. The sound field in the silencer airway is assumed to be a sum of primary and secondary acoustic components. By applying the equivalent surface source method (Namba and Fukushige, 1980), each perforated plate is regarded as a distribution of monopoles generating the secondary sound field. The impedance boundary conditions at the perforated plates are used to relate the secondary sound field with the primary components, so to avoid complex eigenvalue computations. The trigonometric series expansion technique used in the transfer matrix method (Wang and Sun, 2011) is also adopted, which writes the source strength of the distributed monopoles as a function of modal amplitudes of the primary components. The matching conditions, i.e., the continuity of sound pressure and axial particle velocity, is applied in the inlet and outlet planes to calculate the unknowns in the airway, i.e., modal amplitudes of the primary sound field.

\section{METHODOLOGY}

As shown in Fig. 1, a $W \times H$ rectangular duct with one finite porous splitter is considered. The splitter has a length of $L$ and a thickness of $d$. In order to be more realistic, the splitter is terminated at $z=0$ and $z=L$ by a rigid fairing. The inlet and outlet sections are denoted as regions $R_{1}$ and $R_{5}$, respectively. The splitter duct section consists of two airway regions ( $R_{2}$ and $R_{4}$ ) and one porous material region $\left(R_{3}\right)$. These regions are separated by two perforated plates which are located at $x=x_{0}$ and $x=x_{1}$, respectively. In this 
article, the method is established without consideration of mean flow effect but will be further developed to include mean flow in future work.

\section{Sound fields in inlet and outlet sections}

In region $R_{1}$, the sound field can be represented as

$$
\tilde{p}^{R_{1}}=e^{\mathrm{i} \omega t} \sum_{m} \sum_{n}\left[A_{m n}^{R_{1}} e^{\mathrm{i} \gamma_{m n, 1}^{+} z}+B_{m n}^{R_{1}} e^{\mathrm{i} \gamma_{m n, 1}^{-}}\right] \cos \frac{m \pi x}{W} \cos \frac{n \pi y}{H},
$$

where $\mathrm{i}=\sqrt{-1}, A$ and $B$ are the modal amplitudes for incident and reflected sound waves, respectively. $\gamma^{+}$and $\gamma^{-}$are the forward and backward axial wave numbers, respectively. $m$ and $n$ are the mode number in $x$ (perpendicular to perforated plates) and $y$ (parallel to perforated plates) directions, respectively. Here the time dependent $e^{\mathrm{i} \omega t}$ is adopted.

The duct is assumed to be ended by an anechoic termination, so the sound field in region $R_{5}$ is

$$
\tilde{p}^{R_{5}}=e^{\mathrm{i} \omega t} \sum_{m} \sum_{n}\left[A_{m n}^{R_{5}} e^{\mathrm{i} \gamma_{m, 5}^{+}(z-L)}\right] \cos \frac{m \pi x}{W} \cos \frac{n \pi y}{H}
$$
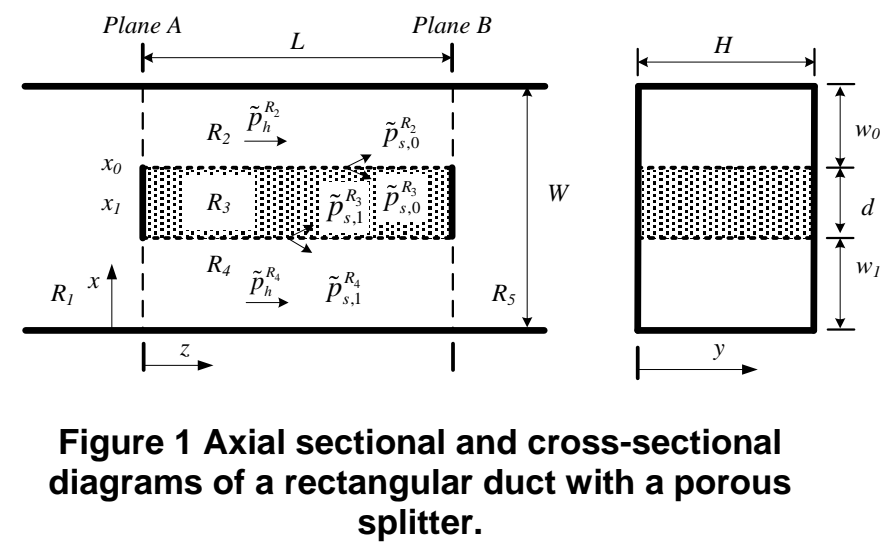

\section{Sound fields in splitter section}

The sound field in airway regions $R_{2}$ and $R_{4}$ is assumed to be a sum of primary and secondary components. The primary component is the sound field that would be realized if the perforated plates separating the porous material and the airway are replaced by sound-hard walls. The perforated plate is modelled as a distribution of monopoles characterized by the normal particle velocity $\tilde{V}_{n}$ of the fluid at the surface of the perforated plate according to the equivalent surface method (Namba and Fukushige, 1980). The equivalent distributed monopoles generate secondary sound waves in both airway regions $\left(R_{2}\right.$ and $\left.R_{4}\right)$ and porous material domain $\left(R_{3}\right)$.

\section{Primary sound components}

Primary sound fields in $R_{2}$ and $R_{4}$ can be easily written as

$$
\begin{aligned}
& \tilde{p}_{h}^{R_{2}}=e^{\mathrm{i} \omega t} \sum_{m} \sum_{n}\left[A_{m n}^{R_{2}} e^{\mathrm{i} \gamma_{m, 2}^{+} z}+B_{m n}^{R_{2}} e^{\mathrm{i} \gamma_{m, 2}^{-} z}\right] \cos \frac{m \pi\left(x-x_{0}\right)}{w_{0}} \cos \frac{n \pi y}{H} \\
& \tilde{p}_{h}^{R_{4}}=e^{\mathrm{i} \omega t} \sum_{m} \sum_{n}\left[A_{m n}^{R_{4}} e^{\mathrm{i} \gamma_{m n, 4}^{+} z}+B_{m n}^{R_{4}} e^{\mathrm{i} \gamma_{m n}^{-}, 4}\right] \cos \frac{m \pi x}{w_{1}} \cos \frac{n \pi y}{H}
\end{aligned}
$$

where $w_{0}$ and $w_{1}$ are the distances from the upper and lower surface of the splitter to nearby duct wall, respectively.

\section{Secondary sound components}

The upper and lower perforated plates are numbered 0 $\left(x=x_{0}\right)$ and $1\left(x=x_{1}\right)$, and the normal particle velocities of the fluid at perforated plates No. 0 and No. 1 are denoted as $\tilde{V}_{n, 0}$ and $\tilde{V}_{n, 1}$. The distributed equivalent monopoles of perforated plate No. 0 radiate secondary sound waves $\tilde{p}_{s, 0}^{R_{2}}$ in region $R_{2}$ and $\tilde{p}_{s, 0}^{R_{3}}$ in region $R_{3}$, while the distributed equivalent monopoles of perforated plate No. 1 generate secondary sound waves $\tilde{p}_{s, 1}^{R_{2}}$ in region $R_{2}$ and $\tilde{p}_{s, 1}^{R_{3}}$ in region $R_{3}$.

By using the Green function method (Goldstein, 1976), the secondary sound fields can be represented as

$$
\begin{aligned}
& \tilde{p}_{s, 0}^{R_{2}}(\boldsymbol{x}, t)=\int_{-T}^{T} \int_{R_{2}} \rho_{0} \tilde{V}_{n, 0}\left(y^{\prime}, z^{\prime}, \tau\right) \delta\left(x^{\prime}-x_{0}\right) \frac{\partial G_{D}^{R_{2}}}{\partial \tau} d V d \tau, \\
& \tilde{p}_{s, 0}^{R_{3}}(\boldsymbol{x}, t)=-\int_{-T}^{T} \int_{R_{3}} \rho_{p} \tilde{V}_{n, 0}\left(y^{\prime}, z^{\prime}, \tau\right) \delta\left(x^{\prime}-x_{0}\right) \frac{\partial G_{C}^{R_{2}}}{\partial \tau} d V d \tau, \\
& \tilde{p}_{s, 1}^{R_{3}}(\boldsymbol{x}, t)=\int_{-T}^{T} \int_{V_{R_{3}}} \rho_{p} \tilde{V}_{n, 1}\left(y^{\prime}, z^{\prime}, \tau\right) \delta\left(x^{\prime}-x_{1}\right) \frac{\partial G_{C}^{R_{3}}}{\partial \tau} d V d \tau, \\
& \tilde{p}_{s, 1}^{R_{4}}(\boldsymbol{x}, t)=-\int_{-T}^{T} \int_{R_{4}} \rho_{0} \tilde{V}_{n, 1}\left(y^{\prime}, z^{\prime}, \tau\right) \delta\left(x^{\prime}-x_{1}\right) \frac{\partial G_{D}^{R_{4}}}{\partial \tau} d V d \tau,
\end{aligned}
$$

where $(\boldsymbol{x}, t)=(x, y, z, t)$ and $(\boldsymbol{y}, \tau)=\left(x^{\prime}, y^{\prime}, z^{\prime}, \tau\right)$ are the coordinates of the observer and the sources, respectively. Here, $G_{D}^{R_{2}}, G_{C}^{R_{3}}$ and $G_{D}^{R_{4}}$ are Green functions in regions $R_{2} \sim R_{4}$, respectively. $\int_{R_{2}} \cdot d V, \int_{R_{3}} \cdot d V$ and $\int_{R_{4}} \cdot d V$ are the volume integrals over regions $R_{2} \sim R_{4}$, respectively. $\delta$ is the Dirichlet function and $\rho_{p}$ is the equivalent density of the porous material. The minus signs in Eqs. (6) and (7) mean the exterior normal of the corresponding source surface points in the negative direction of $x$.

\section{Trigonometric series expansion of normal particle velocity distributions}

As can be seen in Eqs. (5)-(8), the secondary sound components are determined by normal particle velocity distributions at $x=x_{0}$ and $x=x_{1}$. These distributions can be expressed in an easier-to-use form through a double trigonometric series expansion technique as 


$$
\begin{aligned}
& \tilde{V}_{n, 0}\left(y^{\prime}, z^{\prime}, \tau\right)=\sum_{\bar{k}=1}^{K} \sum_{\bar{k}=0}^{N-1} V_{\bar{k} \bar{k}}^{0} \cos \frac{\overline{\bar{k}} \pi y^{\prime}}{H} \sin \frac{\bar{k} \pi z^{\prime}}{L} e^{\mathrm{i} \omega \tau}, \\
& \tilde{V}_{n, 1}\left(y^{\prime}, z^{\prime}, \tau\right)=\sum_{\bar{k}=1}^{K} \sum_{\bar{k}=0}^{N-1} V_{\bar{k} \bar{k}}^{1} \cos \frac{\overline{\bar{k}} \pi y^{\prime}}{H} \sin \frac{\bar{k} \pi z^{\prime}}{L} e^{\mathrm{i} \omega \tau} .
\end{aligned}
$$

Here each series is truncated to an appropriate number. Such a technique was also used in the transfer matrix method (Wang and Sun, 2011). With expressions in Eqs. (9) and (10), the intermediate unknowns of secondary sound components are $V_{\overline{k k}}^{0}$ and $V_{\overline{k k}}^{1}$.

\section{Impedance boundary conditions at perforated plates}

The impedances of the perforated plates No. 0 and No. 1 are denoted as $Z_{s, 0}$ and $Z_{s, 1}$, respectively. The impedance boundary conditions at the perforated plates are

$$
\begin{aligned}
& \frac{\left(\tilde{p}_{h}^{R_{2}}+\tilde{p}_{s, 0}^{R_{2}}\right)-\left(\tilde{p}_{s, 0}^{R_{3}}+\tilde{p}_{s, 1}^{R_{3}}\right)}{\tilde{V}_{n, 0}}=Z_{s, 0}, \quad x=x_{0}, \\
& \frac{\left(\tilde{p}_{s, 0}^{R_{3}}+\tilde{p}_{s, 1}^{R_{3}}\right)-\left(\tilde{p}_{h}^{R_{4}}+\tilde{p}_{s, 1}^{R_{4}}\right)}{\tilde{V}_{n, 1}}=Z_{s, 1}, \quad x=x_{1} .
\end{aligned}
$$

Here $\tilde{V}_{n, 0}$ and $\tilde{V}_{n, 1}$ are also expanded into series like Eqs. (9) and (10) but with coordinates of the observer. These conditions build up the connections between primary and secondary components and can be used to express the intermediate unknowns $V_{\bar{k} \bar{k}}^{0}$ and $V_{\bar{k}}^{1}$ as functions of modal amplitudes $A_{m n}^{R_{2}}, B_{m n}^{R_{2}}, A_{m n}^{R_{4}}$ and $B_{m n}^{R_{4}}$ of primary sound field. To this aim, we adopt a procedure as follows:

(a) substitute Eqs. (1)-(10) into the above two equations;

(b) multiply $\sin \frac{\bar{j} \pi z}{L} \cos \frac{n \pi y}{H}$ on both sides of these equations $(\bar{j}=1,2, \cdots, K, \quad n=0,1, \cdots, N-1)$;

(c) integrate $z$ from 0 to $L$ and integrate $y$ from 0 to $H$.

Performing such a procedure with different $\bar{j}$ and $n$ and making necessary manipulations give

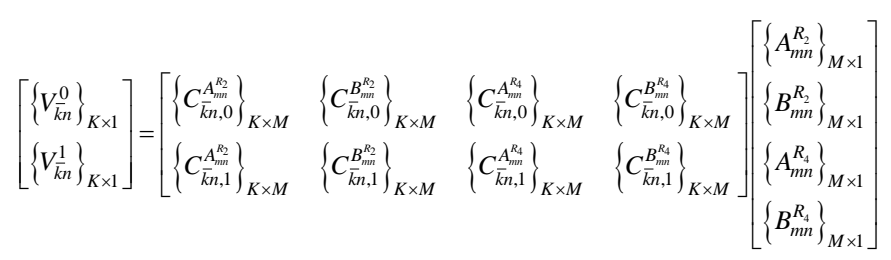

$n=0,1, \cdots, N$

where each $\{\cdot\}$ represents a vector or matrix and the subscript denotes the dimensions. Note $\bar{j}$ disappears in Eq. (13) because this is the final result after matrix inversion and multiplication.

After substituting Eqs. (9), (10) and (13) into Eqs. (5)-(8), the secondary sound components are all expressed by the modal amplitudes $A_{m n}^{R_{2}}, B_{m n}^{R_{2}}, A_{m n}^{R_{4}}$ and $B_{m n}^{R_{4}}$ of primary sound field.

As can be seen above, no eigenvalue computation is encountered and only simple matrix operations are needed.

\section{Mode matching in inlet and outlet planes}

To facilitate illustration, planes $A$ and $B$ are partitioned into several parts as

plane $A \rightarrow\left\{\begin{array}{lll}\text { plane } A_{1}, & y=0 \sim H, & x=x_{0} \sim W \\ \text { plane } A_{2}, & y=0 \sim H, & x=x_{1} \sim x_{0}, \quad z=0 \\ \text { plane } A_{3}, & y=0 \sim H, & x=0 \sim x_{1}\end{array}\right.$
plane $B \rightarrow\left\{\begin{array}{lll}\text { plane } B_{1}, & y=0 \sim H, & x=x_{0} \sim W \\ \text { plane } B_{2}, & y=0 \sim H, & x=x_{1} \sim x_{0}, \quad z=L \\ \text { plane } B_{3}, & y=0 \sim H, & x=0 \sim x_{1}\end{array}\right.$

In the absence of mean flow, the matching conditions become the continuity of sound pressure and axial particle velocity in the inlet and outlet planes, i.e., planes $A$ and $B$. Mathematically speaking,

$$
\begin{aligned}
& \tilde{p}^{R_{1}}=\tilde{p}_{h}^{R_{2}}+\tilde{p}_{s, 0}^{R_{2}},, \boldsymbol{x} \in \text { plane } A_{1}, \\
& \tilde{p}^{R_{1}}=\tilde{p}_{h}^{R_{4}}+\tilde{p}_{s, 1}^{R_{4}}, \boldsymbol{x} \in \text { plane } A_{3}, \\
& \tilde{v}_{z}^{R_{1}}= \begin{cases}\tilde{v}_{z, h}^{R_{2}}+\tilde{v}_{z, s, 0}^{R_{2}}, \boldsymbol{x} \in \text { plane } A_{1} \\
0, & \boldsymbol{x} \in \text { plane } A_{2}, \\
\tilde{v}_{z, h}^{R_{4}}+\tilde{v}_{z, s, 1}^{R_{4}}, & \boldsymbol{x} \in \text { plane } A_{3}\end{cases} \\
& \tilde{p}^{R_{5}}=\tilde{p}_{h}^{R_{2}}+\tilde{p}_{s, 0}^{R_{2}},, \boldsymbol{x} \in \text { plane } B_{1}, \\
& \tilde{p}^{R_{5}}=\tilde{p}_{h}^{R_{4}}+\tilde{p}_{s, 1}^{R_{4}},, \boldsymbol{x} \in \text { plane } B_{3}, \\
& \tilde{v}_{z}^{R_{5}}= \begin{cases}\tilde{v}_{z, h}^{R_{2}}+\tilde{v}_{z, s, 0}^{R_{2}}, & \boldsymbol{x} \in \text { plane } B_{1} \\
0, & \boldsymbol{x} \in \text { plane } B_{2}, \\
\tilde{v}_{z, h}^{R_{4}}+\tilde{v}_{z, s, 1}^{R_{4}}, & \boldsymbol{x} \in \text { plane } B_{3}\end{cases}
\end{aligned}
$$

where $\tilde{v}_{z} \mathrm{~s}$ are the axial particle velocities which can be calculated according to the axial linearized momentum equation

$$
\frac{\partial \tilde{v}_{z}}{\partial t}=-\frac{1}{\rho_{0}} \frac{\partial \tilde{p}}{\partial z}
$$

Because all the eigenfunctions used in this method are those in a hard wall duct, orthogonality is maintained in each region. So, to fully utilize the orthogonality and to assure the accuracy, mode matching is applied. Specifically, multiply $\frac{m \pi\left(x-x_{0}\right)}{w_{0}} \cos \frac{n \pi y}{H} \quad(m=0,1, \cdots, M-1, n=0,1, \cdots, N-1)$ on both sides of Eq. (15) and integrate over plane $A_{1}$; multiply $\quad \cos \frac{m \pi x}{w_{1}} \cos \frac{n \pi y}{H} \quad(\quad m=0,1, \cdots, M-1$, 
$n=0,1, \cdots, N-1)$ on both sides of Eq. (16) and integrate over plane $A_{2}$; multiply $\cos \frac{m \pi x}{W} \cos \frac{n \pi y}{H} \quad(m=0,1, \cdots, 2 M-1$, $n=0,1, \cdots, N-1)$ on both sides of Eq. (17) and integrate over plane $A_{3}$. For Eqs. (18)-(20), similar manipulations are carried out.

From the above deduction, it is noticed that the sound waves with different mode number $n$ (parallel to perforated plates) propagate without interactions, which is easy to understand as the splitter silencer is uniform in $y$ direction. Therefore, the sound attenuation performance of splitter silencer with different mode number $n$ can be accessed separately. With $n$ fixed, the final equation sets can be written as

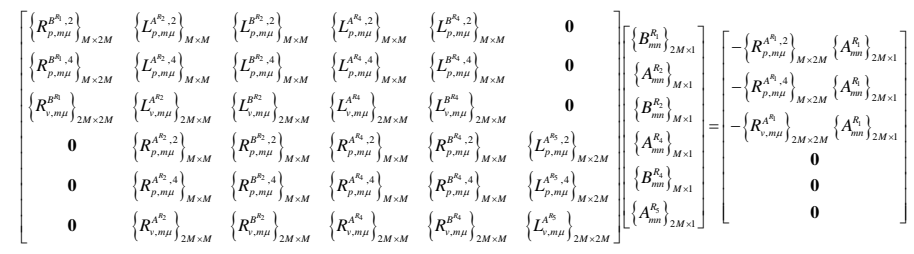

Here, in the inlet or outlet duct section, $2 M$ modes in the $x$ direction are included while in each airway duct of splitter section, $M$ modes are contained in Eq. (22). Since the incident modal amplitudes $A_{m n}^{R_{1}} \mathrm{~s}$ are known, there are $8 M$ unknowns and $8 M$ equations, implying Eq. (22) is closed.

\section{Transmission loss calculation}

The transmission loss $T L$ is defined as

$$
T L=10 \log _{10}\left(\frac{W_{i}}{W_{t}}\right)
$$

where $W_{i}$ and $W_{t}$ are incident and transmitted acoustic power which can be calculated with $A_{m n}^{R_{1}}$ and $A_{m n}^{R_{5}}$ :

$$
\begin{gathered}
W_{i}=\sum_{m=0}^{M_{C}-1}\left|A_{m n}^{R_{1}}\right|^{2} \frac{\Gamma_{m n}\left|\gamma_{m n, 1}^{+}\right|}{2 \rho_{0} c_{0} k_{0}}, \\
W_{t}=\sum_{m=0}^{M_{C}-1}\left|A_{m n}^{R_{5}}\right|^{2} \frac{\Gamma_{m n}\left|\gamma_{m n, 5}^{+}\right|}{2 \rho_{0} c_{0} k_{0}} .
\end{gathered}
$$

Here, $M_{C}$ is the number of cut-on modes in the main duct and $\Gamma_{m n}=\varepsilon_{m} \varepsilon_{n} W H$ where

$$
\varepsilon_{m}=\left\{\begin{array}{ll}
1 & , m=0 \\
\frac{1}{2}, & m \neq 0
\end{array}, \quad \varepsilon_{n}=\left\{\begin{array}{ll}
1 & , n=0 \\
\frac{1}{2} & , n \neq 0
\end{array} .\right.\right.
$$

\section{RESULTS AND DISCUSSION}

In this section, the present method is used to predict the transmission loss of a specific splitter silencer in the absence of mean flow. The geometry of the cross section of the duct is $W \times H=30 \mathrm{~cm} \times 30 \mathrm{~cm}$.The thickness and the length of the splitter is $d=6 \mathrm{~cm}, L=60 \mathrm{~cm}$. The upper surface of the splitter is $w_{0}=12 \mathrm{~cm}$ from the upper duct wall so that $w_{1}=W-w_{0}-d=12 \mathrm{~cm}$. The splitter is filled with porous material, the static flow resistivity of which is $R_{f}=10000$ MKS Rayls/m. The splitter is covered with two perforated plates, the parameters of which are $t_{w}=0.09 \mathrm{~cm}$, $d_{h}=0.249 \mathrm{~cm}$ and $\phi=8 \%$. The Delany-Bazley model for porous material and an impedance model for perforated plates are used, as can be seen in Appendixes A and B. The range of frequency for plane wave incidence is $100 \sim 3000 \mathrm{~Hz}$ and $600 \sim 3000 \mathrm{~Hz}$ for $(0,1)$ and $(1,0)$ mode incidence.

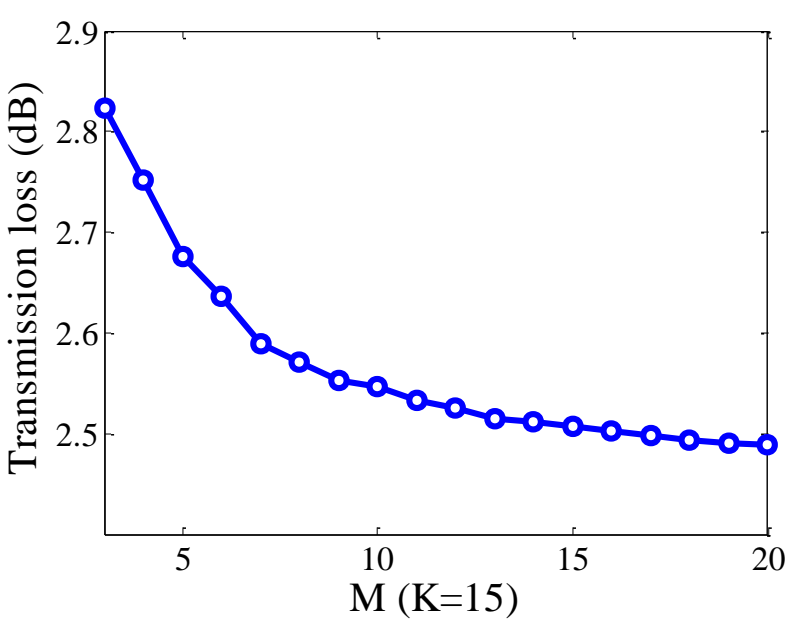

Figure 2 Transmission loss predictions with different $M(f=3000 \mathrm{~Hz}$, plane wave incidence, $K=15)$.

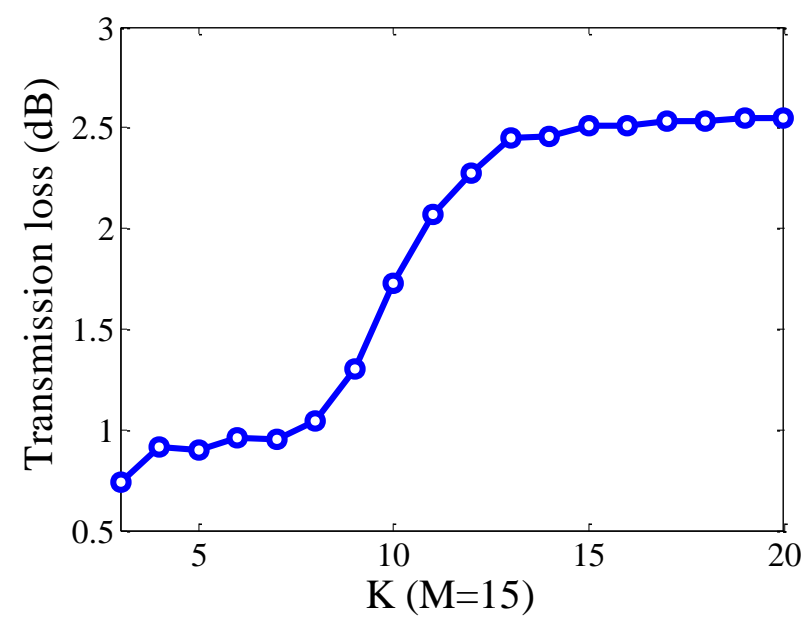

Figure 3 Transmission loss predictions with different $K(f=3000 \mathrm{~Hz}$, plane wave incidence, $M=15)$.

\section{Convergence of the method}

As can be seen in the derivation of the method, the truncated numbers should be chosen appropriately. It is expected that the numbers needs to be larger as the frequency 
$f=\omega / 2 \pi$ increases. So, the convergence of the method is discussed for $f=f_{\max }=3000 \mathrm{~Hz}$. Figures 2 and 3 show the trends of transmission loss predictions for plane wave incidence with different $M$ and with different $K$, respectively. It is seen clearly that the method converges as $M$ and $K$ increase. For higher order mode incidence, similar trends are observed. The values of $M$ and $K$ are set as $M=K=15$ in the following part.

\section{Comparison with FEM}

In order to verify the efficiency and accuracy of the present method, the finite element method (FEM) is also applied with the help of the commercial software COMSOL ${ }^{@}$. The maximum mesh element in FEM simulations is set as $c_{0} / 10 f_{\max }$, denoting the least points per wavelength is 10 .

Figures 4-6 show the comparison of transmission loss predictions by FEM and this article's method. These figures show, for both plane wave and higher order mode incidence, the predicted $T L$ spectra by using this method are in a very good agreement with those by FEM. It is also noticed that $T L$ predicted by this method is slightly higher, which may result from the series truncations.

Compared with FEM, the present method has obvious advantages in computing speed and memory consumption, thus can be used for a large-sized duct in a very high frequency range. Besides, this method is a three dimensional method which can consider higher mode incidence in both $x$ and $y$ directions, which means no additional assumptions are made concerning incident sound waves like in the reduced 2dimensional method (Kirby, 2005, Kirby et al., 2014).

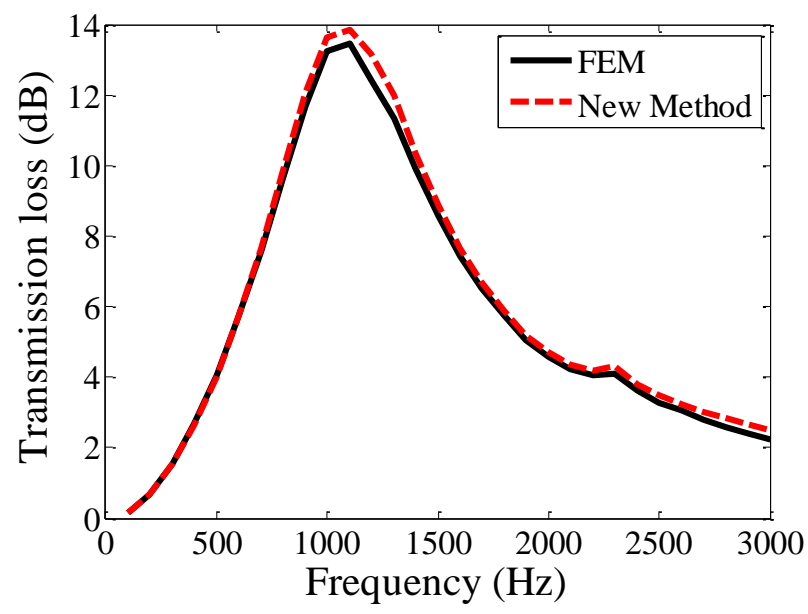

Figure 4 Transmission loss predictions for plane wave incidence by both FEM and this article's method.

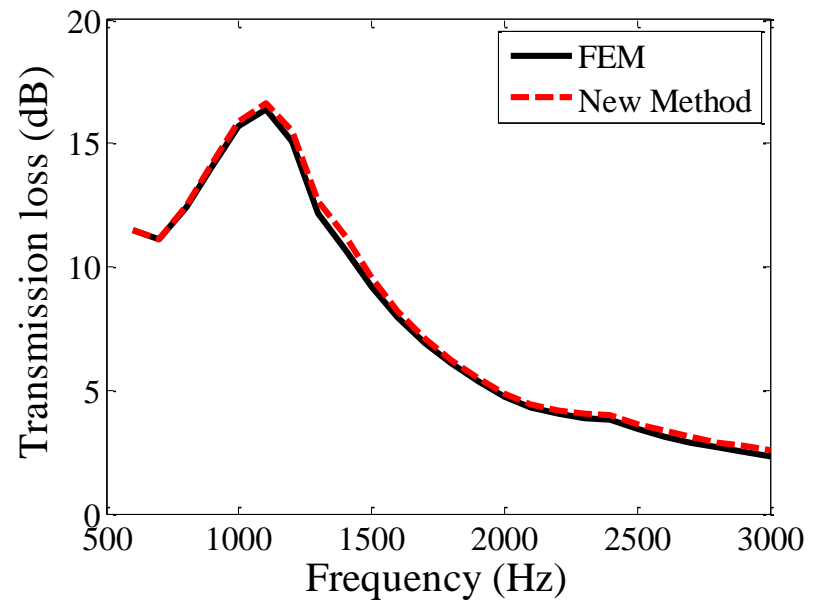

Figure 5 Transmission loss predictions for $(0,1)$ mode wave incidence by both FEM and this article's method.

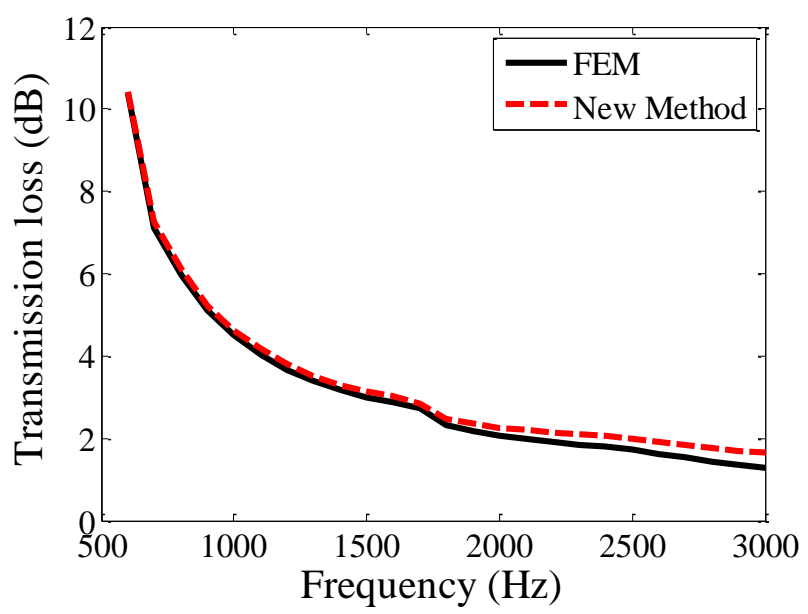

Figure 6 Transmission loss predictions for $(1,0)$ mode wave incidence by both FEM and this article's method.

\section{CONCLUSIONS}

A three-dimensional analytical method is developed to predict the sound attenuation performance of the porous splitter silencer commonly used in a HVAC system. This method includes acoustic scattering effects of either end of the silencer and avoids troublesome eigenvalue calculations. Higher mode incidence in both $x$ and $y$ directions can be considered in this method. For both plane wave and higher order mode incidence, the transmission losses of the splitter silencer predicted by this method and FEM coincide well.

\section{NOMENCLATURE}

$W \quad$ width of the main duct, $m$

$H \quad$ height of the main duct, $\mathrm{m}$

$L \quad$ length of the splitter, $\mathrm{m}$

$d \quad$ thickness of the splitter, $\mathrm{m}$

$w_{0}, w_{1}$ the distances of the upper and lower surfaces of the splitter from the duct wall 
$x_{0}, x_{1}$ the $x$ coordinates of the upper and lower surfaces of the splitter

$\omega$

$f \quad$ frequency, $1 / \mathrm{s}$

$\rho_{0} \quad$ air density, $\mathrm{kg} / \mathrm{m}^{3}$

$c_{0} \quad$ sound speed in air, $\mathrm{m} / \mathrm{s}$

$k_{0} \quad$ wave number in air, $1 / \mathrm{m}$

$\rho_{p} \quad$ equivalent density of porous material

$Z_{s, 0}, Z_{s, 1}$ impedances of perforated plates numbered No. 0 and No. 1

$m, n$ mode numbers in $x$ directions and in $y$ directions

$A, B \quad$ modal amplitude for forward and backward propagating sound wave

$\gamma^{+}, \gamma^{-}$axial wave number for forward and backward propagating sound wave

$R_{i} \quad$ duct region numbered $i$

$\tilde{V}_{n, 0}, \tilde{V}_{n, 1}$ normal particle velocity at the perforated plates numbered No. 0 and No. 1

$V_{\overline{\bar{k}}}^{0}, V_{\overline{k \bar{k}}}^{1}$ expansion coefficients of $\tilde{V}_{n, 0}$ and $\tilde{V}_{n, 1}$

$\tilde{p}^{R_{1}}, \tilde{p}^{R_{5}}$ sound pressure in region $R_{1}$ and $R_{5}$

$\tilde{p}_{h}^{R_{2}}, \tilde{p}_{h}^{R_{4}}$ primary sound component in region $R_{2}$ and $R_{4}$

$\tilde{p}_{s, 0}^{R_{2}}, \tilde{p}_{s, 0}^{R_{3}}$ secondary sound component generated by the perforated plate numbered No. 0

$\tilde{p}_{s, 1}^{R_{2}}, \tilde{p}_{s, 1}^{R_{3}}$ secondary sound component generated by the perforated plate numbered No. 1

$G_{D}^{R_{2}}, G_{C}^{R_{3}}, G_{D}^{R_{4}}$ Green functions in region $R_{2}, R_{3}$ and $R_{4}$

$\tilde{v}_{z} \quad$ axial component of the particle velocity

$M$ the number of truncated series' terms in $x$ direction

$K$ the number of truncated series' terms in $z$ direction

TL transmission loss

$W_{i}, W_{t}$ incident and transmitted sound power

\section{ACKNOWLEDGMENTS}

The research presented here is supported by National Natural Science Foundation of China (Nos. 51676008, 11661141020, 51790514 and 51706006).

\section{APPENDIX A - Delany and Bazley model}

In the Delany and Bazley model (Delany and Bazley, 1970), the porous material is regarded as an equivalent fluid that presents the equivalent complex wave number $k_{p}$ and the characteristic impedance $Z_{p}$ which obey the so-called power law, i.e.,

$$
\begin{aligned}
& k_{p} / k_{0}=1+0.0978 E^{-0.700}-\mathrm{i} \cdot 0.189 E^{-0.595} \\
& Z_{p} / Z_{0}=1+0.0571 E^{-0.754}-\mathrm{i} \cdot 0.087 E^{-0.732}
\end{aligned}
$$

where $E=\rho_{0} f / R_{f}, k_{0}=\omega / c_{0}$ and $Z_{0}=\rho_{0} c_{0}$. Here, $R_{f}$ is the static flow resistivity of the porous material. The equivalent sound speed and density can be calculated as $c_{p}=\omega / k_{p}$ and $\rho_{p}=Z_{p} / c_{p}$. This is an empirical model which is applicable for fibrous porous materials and the effective range of $E$ is $0.01 \sim 1$.

\section{APPENDIX B - Impedance model of the perforated plate}

The acoustic impedance model (Selamet et al., 2001) of perforated plate is used in this paper. The dimensionless acoustic impedance is given by:

$$
\zeta=\left[0.006+\mathrm{i} k_{0}\left\{t_{w}+0.375 d_{h}\left(1+\frac{Z_{p}}{Z_{0}} \frac{k_{p}}{k_{0}}\right)\right\}\right] / \phi
$$

where $\zeta=Z_{s} / Z_{0}, t_{w}$ is the thickness of the plate, $d_{h}$ is the hole diameter and $\phi$ is the porosity.

\section{References}

DELANY, M. E. \& BAZLEY, E. N. 1970. Acoustical properties of fibrous absorbent materials. Applied Acoustics, 3, 105-116.

GOLDSTEIN, M. E. 1976. Aeroacoustics, New York, McGraw-Hill International Book Company.

KIRBY, R. 2005. The influence of baffle fairings on the acoustic performance of rectangular splitter silencers. Journal of the Acoustical Society of America, 118, 2302-2312.

KIRBY, R., AMOTT, K., WILLIAMS, P. T. \& DUAN, W. B. 2014. On the acoustic performance of rectangular splitter silencers in the presence of mean flow. Journal of Sound and Vibration, 333, 6295-6311.

KIRBY, R. \& LAWRIE, J. B. 2005. A point collocation approach to modelling large dissipative silencers. Journal of Sound and Vibration, 286, 313-339.

KO, S. H. 1975. Theoretical analyses of sound attenuation in acoustically lined flow ducts separated by porous splitters (rectangular, annular and circular ducts). Journal of Sound and Vibration, 39, 471-487.

LAWRIE, J. B. \& KIRBY, R. 2006. Mode-matching without root-finding: Application to a dissipative silencer. Journal of the Acoustical Society of America, 119, 2050-2061.

NAMBA, M. \& FUKUSHIGE, K. 1980. Application of the equivalent surface source method to the acoustics of duct systems with non-uniform wall impedance. Journal of Sound and Vibration, 73, 125-146.

SELAMET, A., LEE, I. J., JI, Z. L. \& HUFF, N. T. 2001. Acoustic attenuation performance of perforated absorbing silencers.

WANG, X. Y. \& SUN, X. F. 2011. A new segmentation approach for sound propagation in non-uniform lined ducts with mean flow. Journal of Sound and Vibration, 330, 23692387. 